\title{
BMJ Open Incidence and prevalence of diabetic ketoacidosis (DKA) among adults with type 1 diabetes mellitus (T1D): a systematic literature review
}

\author{
Soulmaz Fazeli Farsani, ${ }^{1}$ Kimberly Brodovicz, ${ }^{2}$ Nima Soleymanlou, ${ }^{3}$ Jan Marquard, ${ }^{4}$ \\ Erika Wissinger, ${ }^{5}$ Brett A Maiese ${ }^{5}$
}

To cite: Farsani SF, Brodovicz K, Soleymanlou N, et al. Incidence and prevalence of diabetic ketoacidosis (DKA) among adults with type 1 diabetes mellitus (T1D): a systematic literature review. BMJ Open 2017;7:e016587. doi:10.1136/ bmjopen-2017-016587

- Prepublication history and additional material are available online. To view these files, please visit the journal online (http://dx.doi.org/10.1136/ bmjopen-2017-016587).

Received 24 February 2017 Revised 16 June 2017 Accepted 22 June 2017

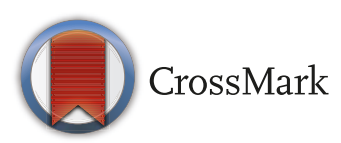

${ }^{1}$ Corporate Department GlobalEpidemiology, Boehringer Ingelheim International GmbH, Ingelheim am Rhein, Germany ${ }^{2}$ Global Epidemiology, Boehringer Ingelheim Pharmaceuticals Inc., Ridgefield, USA

${ }^{3}$ Boehringer Ingelheim Canada, Burlington, Canada

${ }^{4}$ Boehringer Ingelheim Pharma GmbH \& Co KG, Ingelheim am Rhein, Germany

${ }^{5}$ Xcenda L L C, Palm Harbor, Florida, USA

Correspondence to Dr Soulmaz Fazeli Farsani; soulmaz.fazeli_farsani@ boehringer-ingelheim.com

\section{ABSTRACT}

Objectives To summarise incidence and prevalence of diabetic ketoacidosis (DKA) in adults with type 1 diabetes (T1D) for the overall patient population and different subgroups (age, sex, geographical region, ethnicity and type of insulin administration).

Design Systematic literature review (SLR).

Data sources Medline (via PubMed) and Embase (1 January 2000 to 23 June 2016).

Study selection Peer-reviewed observational studies with reported data on the incidence or prevalence of DKA in T1D adults were included. A single reviewer completed the study screening and selection process and a second reviewer performed an additional screening of approximately $20 \%$ of the publications; two reviewers independently conducted the quality assessment; the results were narratively synthesised.

Results Out of 1082 articles, 19 met the inclusion and exclusion criteria, with two additional studies identified that did not specify the patient age range and are therefore not included in the SLR. Overall, eight studies reported incidence with a range of 0-56 per 1000 person-years (PYs), with one outlying study reporting an incidence of 263 per 1000 PYs. Eleven studies reported prevalence with a range of 0-128 per 1000 people. Prevalence of DKA decreased with increasing age. Subgroup analyses were performed using data from no more than two studies per subgroup. There was a higher prevalence of DKA reported in women, non-whites and patients treated with insulin injections compared with men, whites and patients using continuous subcutaneous insulin infusion pumps, respectively.

Conclusions To our knowledge, this is the first SLR on the epidemiology of DKA in T1D adults. Despite an increasing prevalence of T1D in recent years, DKA in adults has been poorly characterised. In an era when the benefit-risk profiles of new antidiabetic therapies are being evaluated, including the potential risk of DKA, there is a clear need to better elucidate the expected rate of DKA among T1D adults.

\section{INTRODUCTION}

Diabetes is a disease characterised by high blood glucose resulting from abnormal insulin production, function or both. ${ }^{1}$ Type
Strengths and limitations of this study

- To our knowledge, this is the first literature review to systematically assess and summarize the incidence rate and prevalence of diabetic ketoacidosis (DKA) in adults with type 1 diabetes (T1D).

- Both young adults and the elderly were included in this systematic literature review (SLR), so the results may be applicable across a wide spectrum of adult patients with T1D.

- The quality of included studies was assessed using a standardised tool (the Joanna Briggs Institute prevalence studies quality assessment tool)

- This review, like any SLR, is subject to publication bias, as an SLR inherently relies upon data available in the published literature.

- Studies not published in English were excluded from the SLR, as were studies of fewer than 50 patients.

1 diabetes mellitus (T1D) is an autoimmune disease caused by the immune-mediated destruction of pancreatic beta cells. ${ }^{1}$ This destruction is modulated by the body's immune system and leads to a limitation in, or complete cessation of, the production and secretion of insulin, which results in the need for external insulin delivery for survival. ${ }^{1}$ T1D typically follows an acute clinical course, with patients presenting with polyuria, polydipsia and weight loss. ${ }^{2}$ According to the International Diabetes Federation, approximately 542000 children $0-14$ years of age have T1D, with 86000 new cases diagnosed worldwide each year. ${ }^{3}$ While there are geographical differences, the overall annual increase in the incidence of T1D is estimated at approximately $3 \%-4 \% .{ }^{34}$ Diagnosis of T1D typically occurs in childhood; in the USA, the peak (mean) age at diagnosis is approximately 14 years.

Information regarding the epidemiology of T1D specifically in adults is scarce; many 
epidemiological studies of adult patients categorise those with blood glucose levels above a certain threshold as simply diabetic, without providing more detailed data on the relative proportions of patients with T1D versus type 2 diabetes mellitus (T2D). ${ }^{3}$ Approximately 5\% of adult-diagnosed cases of diabetes are diagnosed as T1D, ${ }^{1}$ although an Italian study has shown rates of T1D as high as $50 \%$ of incident cases of diabetes among normalweight adults (aged 30-54 years). ${ }^{5}$ Incidence of T1D varies by age and geographical location, ranging from 4.9 per 100000 people in Austria to 61.7 per 100000 people in the USA. ${ }^{6-9}$ A recent systematic literature review (SLR) reported the incidence of T1D to be 1.5 times higher in males than in females less than 40 years of age. ${ }^{6}$

Diabetic ketoacidosis (DKA) is a major acute metabolic complication of T1D that is typically marked by acidosis, ketosis and usually hyperglycaemia. ${ }^{10-12}$ The symptoms of uncontrolled diabetes that may lead to development of DKA are typically of short duration and include polyuria, polydipsia, polyphagia, weight loss, vomiting, abdominal pain and fatigue. ${ }^{10-12}$ Diabetic ketoacidosis is diagnosed in different ways, but typically the following three factors are present: elevated plasma glucose $(>250 \mathrm{mg} / \mathrm{dL})$, ketones in serum or urine and acidosis (serum bicarbonate $<18 \mathrm{mEq} / \mathrm{L}$ and/or $\mathrm{pH}<7.30) .{ }^{10}$ Management of DKA includes fluid and electrolyte therapy, insulin therapy, treatment of any identified triggering causes (eg, continuous subcutaneous insulin infusion (CSII) pump failure, sepsis, pneumonia, acute pancreatitis, cerebrovascular accident, myocardial infarction, stroke, trauma, medications that affect carbohydrate metabolism) and monitoring of therapy and resultant complications. ${ }^{10} 1213$ Excessively rapid fluid resuscitation should be avoided to prevent cerebral oedema, a rare but debilitating and potentially fatal complication of DKA. ${ }^{10}$ While inpatient mortality rates for DKA are generally very low $(<1 \%$ in Scotland ${ }^{14}$ and in the $\mathrm{USA}^{15}$ rates vary substantially based on healthcare setting; a recent analysis conducted in India reported that up to $30 \%$ of hospitalised DKA cases result in inpatient death. ${ }^{13}$ Among all T1D-related deaths for patients aged less than 30 years, $54 \%-76 \%$ can be attributed to DKA. ${ }^{16-19}$ Risk factors associated with a higher frequency of DKA may include younger age at time of DKA hospitalisation, ${ }^{2021}$ higher mean glycosylated haemoglobin A1c (HbAlc), ${ }^{11}{ }^{20-23}$ infection, ${ }^{24}$ CSII pump failure, ${ }^{25} 26$ lower socioeconomic status/household income, ${ }^{11} 2022$ lower physical activity level ${ }^{27}$ and psychiatric symptoms/depression. ${ }^{20} 2829$ The prevalence of DKA at initial disease presentation in paediatric patients with T1D is well documented ${ }^{3031}$; however, information on the prevalence or incidence in adults is limited. One study using the T1D Exchange Clinic Registry in the USA found that $4.8 \%$ of participants reported one or more DKA events (requiring self-reported overnight hospitalisation) in the previous 12 months. ${ }^{11}$ The objectives of this SLR were to summarise the available epidemiological data (incidence rate and prevalence) for DKA in adult patients (aged $\geq 18$ years) with T1D from population-based and other observational studies and to evaluate trends in the evidence for the overall patient population and specific subgroups (age, sex, geography, ethnicity and type of insulin administration such as CSII or multiple daily injections (MDIs)).

\section{METHODS}

\section{Search strategy and selection process}

This systematic review was prepared according to the Preferred Reporting Items for Systematic reviews and Meta-Analysis guidelines. ${ }^{32}$ The study protocol (including search strategy and search terms) was developed and accepted by all authors prior to commencement of the search and can be found in online supplementary appendix 1. Medline (via PubMed) and Embase databases were searched for articles published between 1 January 2000 and 23 June 2016 (date of search execution) by a single review author (EW). The searches were filtered for human studies, but no language restrictions or geographic limitations were applied to the search strategy. Note that only studies published in English were included. Relevant studies published in other languages were noted during screening and a list of these citations reviewed for consideration; it is of note that fewer than 10 non-English studies which had English abstracts available for review were identified and none were deemed relevant for inclusion based on the abstracts. Included studies were peer reviewed, and ongoing studies without peer-reviewed publication (eg, conference abstracts) were excluded. The search results were combined in a referencing software database, and duplicate records were removed.

On second-pass review, a single review author (EW or BAM) applied the following criteria. The targeted population was men and women aged at least 18 years with T1D. When sufficient data were available in the publications to permit determination of the patients' age range (for example, mean age and SD (SD) or SE), the relevant calculations were performed to determine eligibility for inclusion (for example, mean age \pm three SD as an estimate of the range of study patients' age). If the mean age was reported and the calculated age for minus three SDs was $<18$ years, the study was excluded, unless the study population was explicitly described as adults aged $\geq 18$ years. Studies of mixed populations (paediatric and adult patients and/or T1D and T2D) were included only if stratified data were reported for adult patients with T1D . This review was not restricted by specific interventions or comparators and included all patients with T1D , regardless of treatment. DKA outcomes were considered only for patients who were previously identified as having T1D; publications reporting data only for DKA episodes at presentation or diagnosis of T1D were excluded. Type of insulin and method of administration were of interest and were captured when these data were available. Peer-reviewed population-based observational studies, SLRs and meta-analyses of human studies were included. This SLR 
was originally intended to include only population-based studies but was expanded to include other observational study designs such as clinic-based and (potentially unrepresentative) registry studies since there was limited population-based studies in the peer-reviewed literature. Because the aim of the review was to assess real-world epidemiology reported in the peer-reviewed literature describing studies of humans, randomised controlled trials/clinical trials and interventional studies were excluded, as were preclinical or animal studies, editorials, letters, commentaries, case studies, reports or case series, theses and dissertations, narrative literature reviews, small studies (sample size of fewer than 50 patients), guidelines and unpublished studies (eg, conference abstracts).

\section{Data extraction and analysis}

Data extraction was conducted by a single review author (EW or BAM) using a Microsoft Excel file with standardised definitions for each data element that was extracted from each study (see online supplementary appendix 1). Data of interest were incidence rate of DKA (number of new DKA events out of accumulated patient time under study, in person-years (PYs)) and prevalence of DKA (number of DKA events among the total number of patients at risk). Data on risk factors and clinical parameters associated with DKA events were also extracted when reported in the included publications. When sufficient data were available in the published literature to allow computation of these outcomes if not specifically reported by the authors (for example, the number or proportion of patients who experienced a DKA event over a defined time period), the appropriate calculations were performed and are noted as such. For two publications, cumulative incidence was calculated (number of new DKA events out of total patients at risk) because data on incidence rates were not directly reported in the publications. Results from data extraction, including incidence rate, prevalence and risk factors for DKA can be found in online supplementary appendix 2 and supplementary appendix 3 .

The quality of included studies was assessed by a trained epidemiologist (BAM), with consideration of the study design, disease ascertainment, response rate (if applicable), definition of DKA, representativeness of the study population and major potential biases. A table describing these factors for each included study can be found in this report (table 1). Additionally, a standardised quality assessment tool, the Joanna Briggs Institute (JBI) Critical Appraisal Tools 'Checklist for Prevalence Studies' (available at http://joannabriggs.org/research/criticalappraisal-tools.html), was selected as an appropriate tool for assessment of the included study designs. Quality assessment of each included study using the JBI Checklist for Prevalence Studies was undertaken independently by two reviewers (EW and BAM), with any discrepancies resolved by a third reviewer (Xcenda employee, member of evidence synthesis team). The results of the quality assessment using the JBI tool can be found in online supplementary appendix 3 .

\section{Patient involvement}

No patients were involved in setting the research question, in developing plans for design, interpretation, reporting or implementation of the study. No patients were asked to advise on interpretation or reporting of results. There are no plans to disseminate the results of the research to patient communities.

\section{RESULTS}

summarises the literature review process, including the number of records identified, the screening and eligibility results and the final list of included references. Out of 1082 articles identified through the initial search, 19 peer-reviewed observational study publications met the inclusion and exclusion criteria; no SLRs or meta-analyses were identified that met the inclusion and exclusion criteria. There were 11 publications in North America (USA and Canada), ${ }^{11} 20-22$ 29 33-38 five publications in Europe ${ }^{27} 28$ 39-41 and two publications in Israel and one publication in China. ${ }^{23} 4243$ Overall, eight studies reported incidence rate, with a range of 0-263 per 1000 PYs, ${ }^{23} 27283435394243$ and 11 studies reported prevalence with a range of 0-128 per 1000 people. ${ }^{11}$ 20-22 $293336-384041$ The lowest incidence rates were reported in Israel and North America (both 0 events per $1000 \mathrm{PYs})^{34} 43$ and the highest in China (263 events per $1000 \mathrm{PYs})^{42}$ (figure 2). The lowest prevalence was reported in Sweden $(0 \text { per } 1000 \text { people })^{41}$ and the highest in Canada (127.9 per 1000 people) ${ }^{21}$ (figure 3A).

In terms of baseline characteristics, patient selection and descriptions of outcomes, there were some broad similarities across studies included in this review. In most studies, specific definitions or diagnostic criteria for T1D were not described, and some studies did not fully report patient baseline demographic information such as patient ethnicity or insulin delivery method. Standardised measurements of DKA events were not frequently used, as many studies (seven of 19) relied on patient self-report of DKA episodes or hospitalisation records (four of 19). Three publications ${ }^{11} 2229$ using data from the T1D Exchange Clinic registry directly compared DKA data as identified by patient self-reported and clinic-documented events and found that, while the frequency of participant-reported DKA events was higher than clinic-reported events based on medical records, results from logistic regression models designed to assess potential associations with DKA episodes were similar for both sets of data. ${ }^{11}$ Few exclusion criteria were applied to the patient populations, most commonly pregnancy and lack of available data. The age range of patient cohorts varied across studies, with some investigations (2 of 19) restricted to young adults only (approximately 18-26 years of age), four studies focused on adults aged approximately 20-55 years and most (12 studies) evaluating adults of all ages, including those aged over 65 years. Most (16 of 19) studies had approximately a 1:1 male-tofemale ratio of patients. Ethnicity of the patient cohorts was only reported in eight studies; when reported, the 


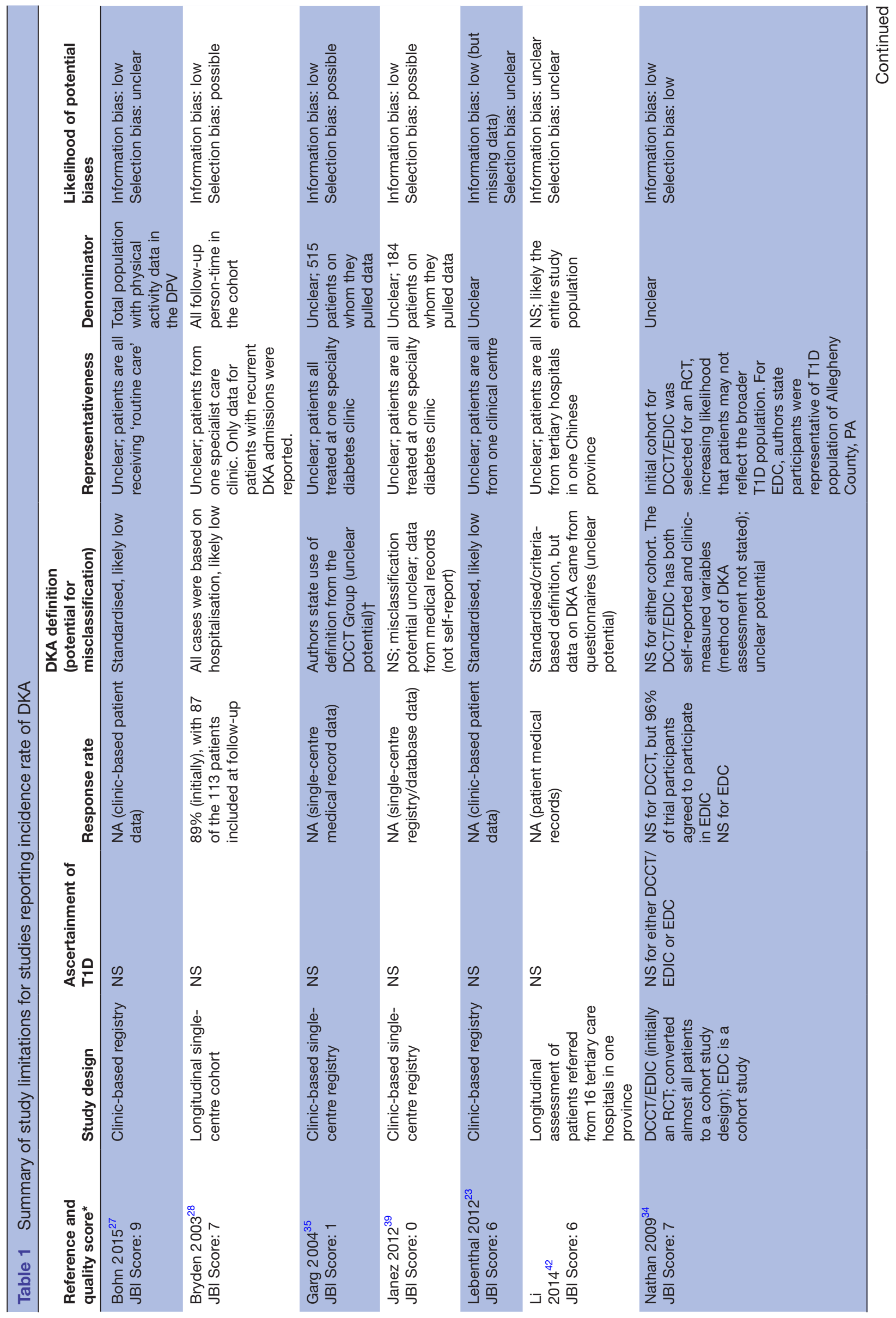




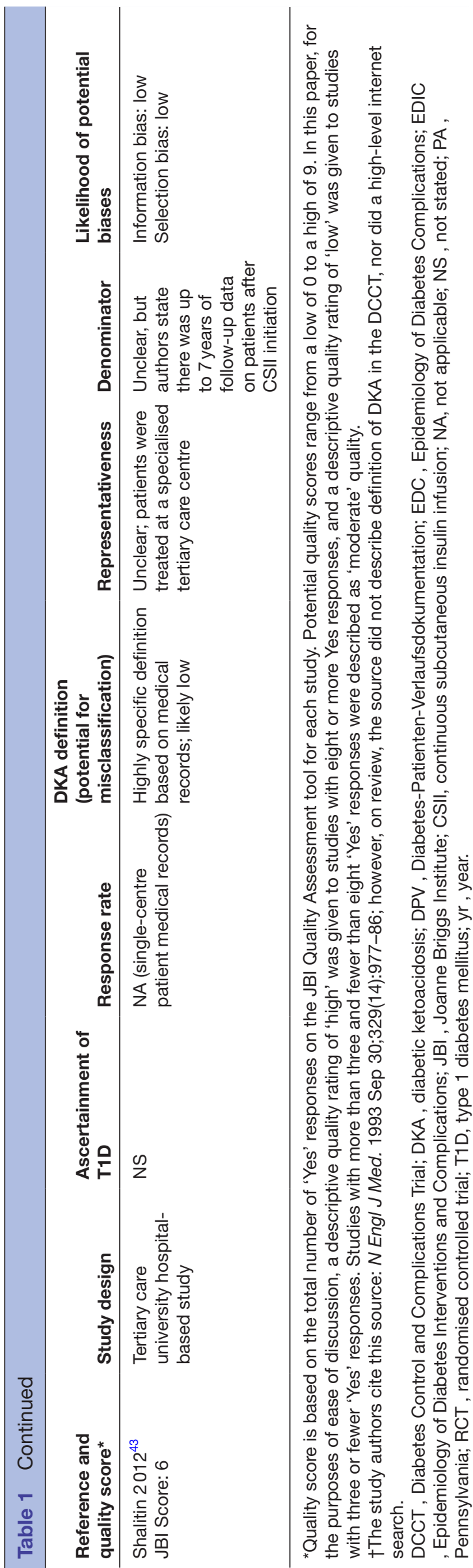

vast majority of patients $(>80 \%)$ were of white non-Hispanic ethnicity. In the publications providing data on insulin delivery method in unselected populations (11 of 19 studies), most $(50 \%-60 \%) \%)$ patients were treated with CSII. While the number of studies providing data on insulin delivery method was limited, there seemed to be an overall trend towards an increasing proportion of CSII users in more recent publications/study periods compared with older investigations.

\section{Overall incidence and prevalence of DKA in North America}

Eleven studies conducted in North America (USA and Canada) reported incidence rate (2) or prevalence (9) for DKA events in adults with T1D (see online supplementary appendix 2). Results from two long-term observational cohorts found that the incidence of DKA showed a general reduction over the duration of study follow-up, with an incidence rate of approximately 20 cases per 1000 PYs at baseline to 0 events at the 12 year follow-up in one cohort and a decrease from approximately 30 cases per 1000 PYs at baseline to $<10$ cases per 1000 PYs at the 18 year follow-up in another cohort. ${ }^{34}$ A US-based study from a single clinic in Colorado compared CSII pump users (42\% of patients) with patients treated with MDIs (58\% of patients),$^{35}$ over the study period of approximately 1 year, only patients treated with CSII experienced any DKA events. In this investigation, the cumulative incidence of DKA was calculated to be 55.6 per 1000 people for CSII users.

All of the publications describing prevalence of DKA in the USA were based on data from the T1D Exchange Clinic Registry, although each investigation evaluated a slightly different patient population. All of these studies relied on patient self-report to determine occurrence of DKA events, and the recall period varied from three to 12 months. For most studies of the larger patient population from the registry, prevalence ranged from approximately 50 to 100 per 1000 people. ${ }^{11} 223338$ Slightly higher DKA prevalence than that reported in the US-based studies was observed in two studies from Canada, both of which linked data from the following databases to determine DKA prevalence: Diabetes, Hypertension and Cholesterol Centre in Calgary; Alberta Inpatient Discharge Abstract Database; Alberta Kidney Disease Network and Statistics Canada 2006 Census Data. ${ }^{20} 21$ In these linked database studies, calculated prevalence was found to be 103 per 1000 people ${ }^{21}$ and 128 per 1000 people. $^{20}$ These Canadian studies relied on linked data from hospital inpatient admissions rather than patient self-report.

\section{Overall incidence and prevalence of DKA in Europe}

In very broad terms, the incidence rates and prevalence reported for European studies (see online supplementary appendix 2) were similar to those described in investigations conducted in North America; however, these trends should be considered with substantial caution given the very small number of European publications reporting each outcome. Only five publications described the 


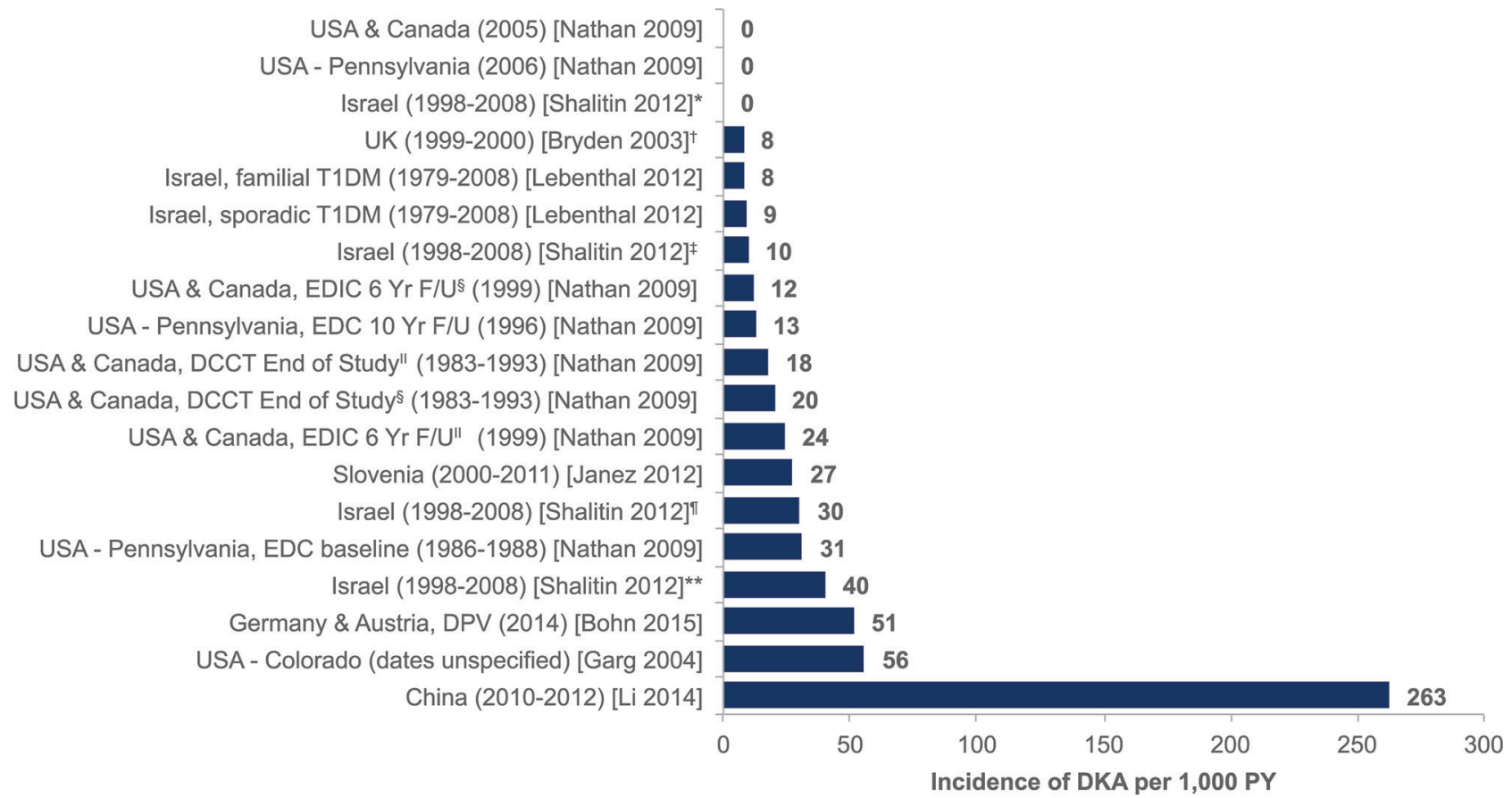

Figure 2 Incidence rate per 1000 PY of DKA in adults with T1D (reported in eight studies). Submitted as image. Footnotes. ${ }^{*}$ Calculated value based on data contained within publication. †Patients who initiated CSII within 1 year of diagnosis, aged $>19$ years at CSII initiation. ¥Patients who initiated CSII within 1 year of diagnosis, aged $>19$ years at last visit. "Conventional treatment arm from DCCT. §Intensive treatment arm from DCCT. ๆPatients who initiated CSIl at least 1 year postdiagnosis, aged $>19$ years at CSII initiation. ${ }^{*}$ Patients who initiated CSII at least 1 year postdiagnosis, aged $>19$ years at last visit. CSII, continuous subcutaneous insulin infusion; DCCT, Diabetes Control and Complications Trial; DKA, diabetic ketoacidosis; DPV, Diabetes-Patienten-Verlaufsdokumentation; EDC, epidemiology of diabetes complications; EDIC, epidemiology of diabetes interventions and complications; F/U, follow-up; PY, person-years; T1D, type 1diabetes mellitus; UK, United Kingdom; USA, United States of America; yr, year.

epidemiology of DKA in adult patient populations in Europe; two reported on patients from the Diabetes-Patienten-Verlaufsdokumentation (DPV) database, which includes patients in Germany and Austria ${ }^{27} 40$ and three evaluated small single-centre patient cohorts. ${ }^{28} 3941$ In all cases, DKA events were ascertained based on patient medical records.

Incidence rates ranged from approximately 8 cases per 1000 PYs (calculated rate based on hospital admissions for DKA) in a study of 113 young adults in Oxfordshire, England ${ }^{28}$ to 51.3 cases per 1000 PYs (reported rate based on $\mathrm{pH}<7.3$ or hospital admission for DKA) for over 18000 adult patients selected from the DPV database. ${ }^{27}$ A Slovenian single-centre study of patients treated with $\mathrm{CSII}^{39}$ provided sufficient data to calculate cumulative incidence of 27.2 cases per 1000 , a lower value than that observed in a similar US-based study (55.6 per 1000). ${ }^{35} \mathrm{It}$ should be noted that while the study period was unclear in the US investigation, based on publication date (2004 for the US study), it is likely that the Slovenian study (with a study period through 2011) included more recent data. This may have had an impact on DKA incidence given the technological improvements in insulin pumps and glucose monitoring over the duration of the Slovenian study (2000 through 2011, and patients had to have 3 or more years of complete data to be included in this study); indeed, the US study reported that the majority of DKA events recorded were due to pump malfunctions.

Prevalence of DKA was reported in two European studies. ${ }^{40}{ }^{41}$ One single-centre longitudinal study of 104 patients performed in Sweden ${ }^{41}$ found a numerical (but not statistically significant) reduction in DKA cases with increasing year of age from 18 to 24 years (prevalence ranged from 0 to 60 cases per 1000 people over this period and was calculated for each 1 year of age separately by the authors). A cross-sectional analysis of patients from the DPV database examining the association of variability in basal insulin rates with various outcomes reported the prevalence of DKA as 39 cases per 1000 people, ${ }^{40}$ also a slightly lower value than the prevalence data reported for US-based or Canadian-based studies.

\section{Overall incidence of DKA in other regions}

Three studies conducted in other regions reported only incidence rate data (see online supplementary appendix 2 ), with very low rates observed in two studies based on patient medical records from the same tertiary care facility in Israel $^{23} 43$ and very high rates described in a multicentre study conducted in tertiary care units in a single province in China. ${ }^{42}$ The low incidence rates in the Israeli studies (ranging from 0 to 40 cases per $1000 \mathrm{PY}$ ) may reflect some selection bias in the patient population 
A. Overall

USA, HbA1c <6.5\% (2010-2012) [Simmons 2013]

USA, $\geq 26$ yrs of age* $(2010-2012)$ [Wong 2014] ${ }^{\dagger}$

USA, $\geq 26$ yrs of age ${ }^{\ddagger}$ (2010-2012) [Wong 2014]

USA (2013-2014) [Miller 2015]

Sweden, 20 yrs of age (1996-2006) [Sparud-Lundin 2008]

Sweden, 19 yrs of age (1996-2006) [Sparud-Lundin 2008]

Germany \& Austria (1995-2014) [Laimer 2016]

USA, 18 to $<26$ yrs of age* $(2010-2012)$ [Wong 2014] USA, not depressed (2010-2012) [Trief 2014]

USA (2010-2012) [Weinstock 2013]

Sweden, $18 \mathrm{yrs}$ of age (1996-2006) [Sparud-Lundin 2008]

USA, 18 to $<26$ yrs of age ${ }^{\ddagger}(2010-2012)$ [Wong 2014]

USA, overall (2010-2012) [Beck 2012]

USA, definite T1DM (2010-2012) [Beck 2012] USA (2010-2012) [Cengiz 2013]

Canada (2004-2009) [Butalia 2013] $]^{\dagger}$

USA, depressed (2010-2012) [Trief 2014]

USA, HbA1C $\geq 8.5 \%$ (2010-2012) [Simmons 2013]

Canada (2004-2008) [Butalia 2014]

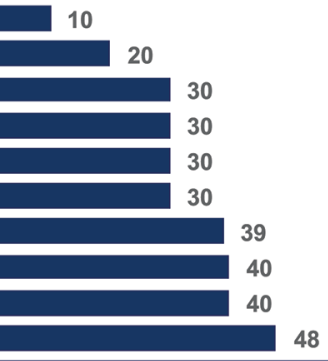

48

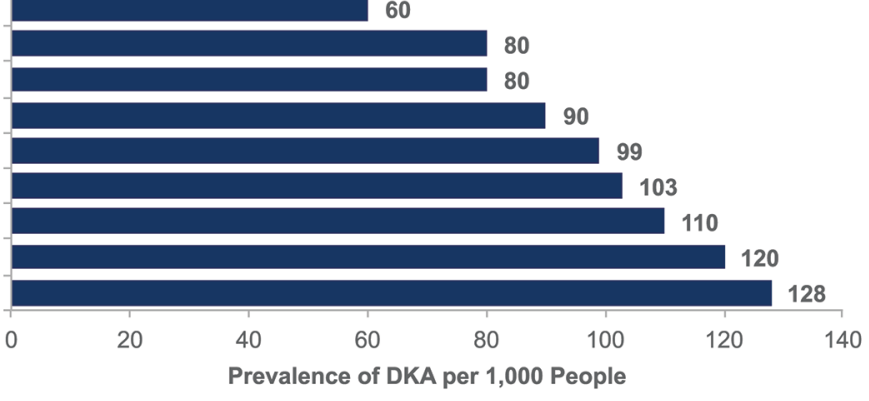

\section{B. By Subgroups of Interest}

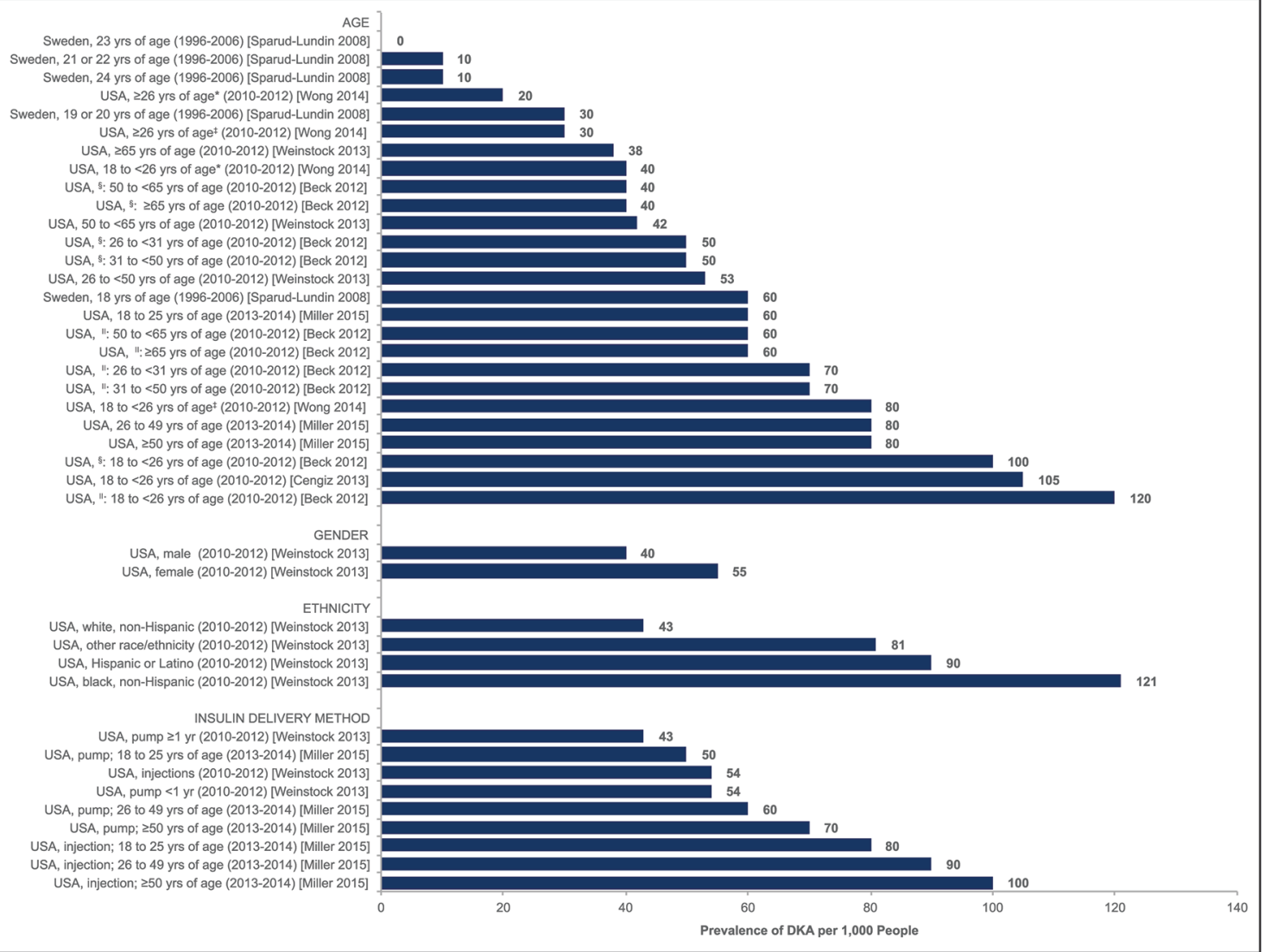

Figure 3 Prevalence (per 1000 people) of DKA in adults with T1D (reported in 11 studies). Submitted as image. Footnotes. ${ }^{*} \mathrm{CGM}$ non-user. †Calculated value based on data contained within publication. $¥ \mathrm{CGM}$ user. §Overall study population. IDefinite T1D. CGM, continuous glucose monitoring; CSII, continuous subcutaneous insulin infusion; DKA, diabetic ketoacidosis; HbA1c, glycosylated haemoglobin; MDI, multiple daily injection; NR, not reported; T1D, type 1 diabetes mellitus; yrs, years.

treated at this single centre. ${ }^{2343}$ In the Chinese study, ${ }^{42}$ the authors acknowledge that the incidence rate observed in their study (263 per $1000 \mathrm{PY}$ ) is considerably higher than values cited in published reports from other countries. The investigators attribute this discrepancy, at least in part, to differences in national healthcare systems, which 


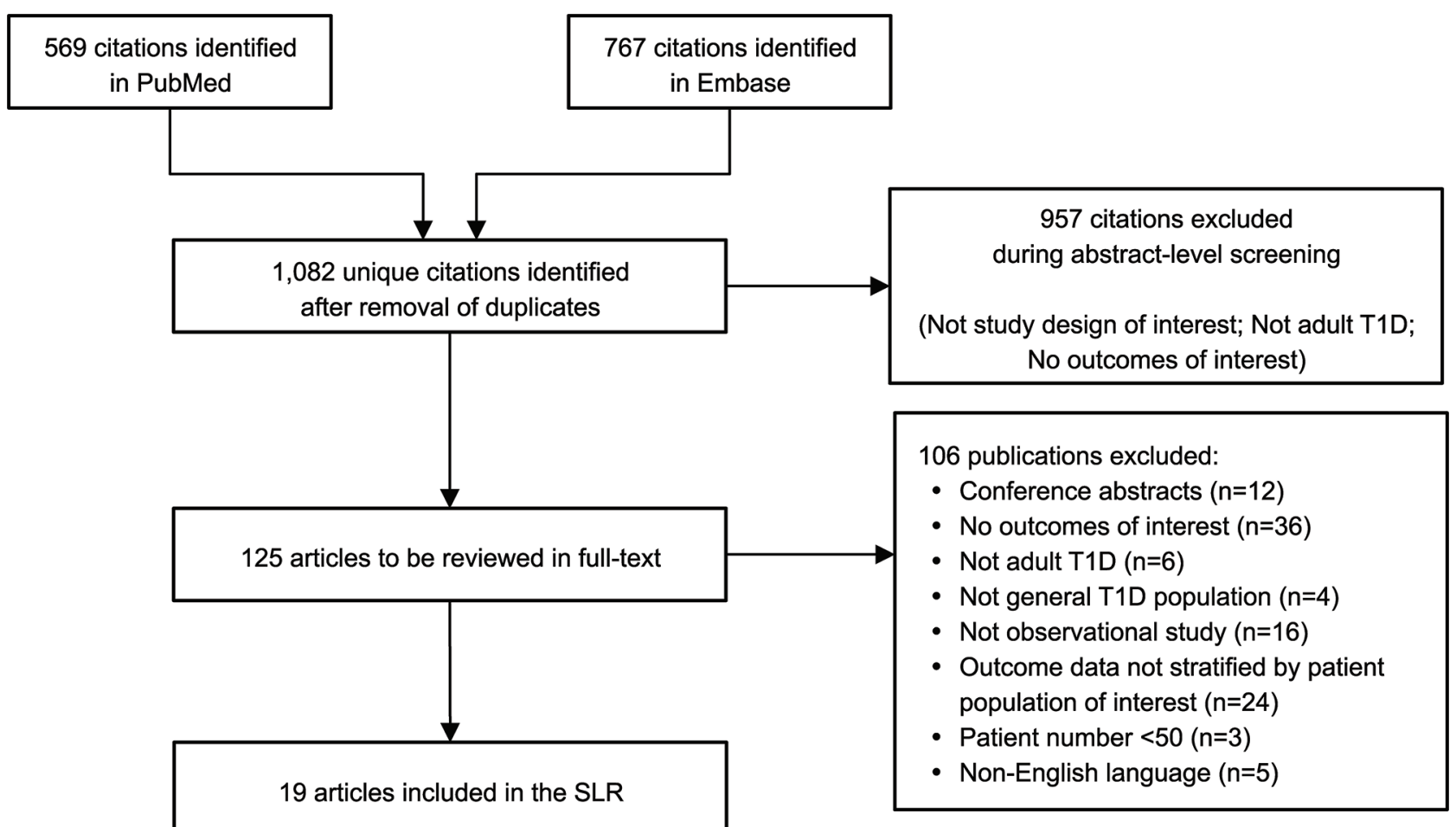

Figure 1 Literature selection and review process. Submitted as image. Footnotes. Key search terms: Type 1 diabetes; adult; diabetic ketoacidosis. SLR, systematic literature review; T1D, type 1 diabetes mellitus.

may limit access to routine healthcare for some patients with T1D in China, as well as infrequent self-monitoring of blood glucose by patients and inappropriate treatment or errors in diabetes management. Although not explicitly described in the publication, it seems likely that this study reported the incidence rate of all DKA events during the study period rather than only the first instance of DKA for each patient. The authors stated that in this study, more than a third (34.4\%) of DKA events represented recurrent (two or more) episodes of DKA for 3.8\% of patients, suggesting that a small population of very high-risk patients contributed substantially to the overall incidence rate.

\section{DKA prevalence by age}

Five publications from the US-based T1D Exchange Clinic Registry ${ }^{11} 22333638$ reported prevalence of DKA among adults with T1D with outcome data stratified by age, as did one study conducted in Sweden and described previously in the 'Overall incidence and prevalence of DKA in Europe' subsection (figure 3B). ${ }^{41}$ In the US-based studies, four of the studies examined a broad sample of adult patients aged 18 to $>90$ years; one analysis ${ }^{22}$ focused solely on young adults. Given the design of the registry, all five studies relied on patient self-report of past DKA events; three publications ${ }^{11} 2233$ examined the prior 12-month period and two ${ }^{36}$ queried patients about the previous 3 months. There was a general trend of decreasing DKA prevalence with increasing age observed across most studies providing age-stratified data. Young adults (aged 18 years to 25 years) had the highest prevalence of DKA (100-120 cases per 1000 in studies with 12-month recall and 40-80 cases per 1000 in studies with 3-month recall), while the elderly (aged $\geq 65$ years) had the lower prevalence of DKA (38-60 cases per 1000 in studies with 12-month recall). The only exception to this trend was a study in which prevalence of DKA was similar across all three adult age ranges (18-25 years, $26-49$ years and $\geq 50$ years). ${ }^{36}$ The DKA data in this particular investigation may have been affected by the study requirement that all patients had an annual follow-up visit at which HbAlc was measured and the shorter duration of recall for DKA events (3 months). There was no information on incidence rate stratified by patient age reported in any studies identified by this SLR.

\section{DKA prevalence by other patient subgroups of interest}

Subgroup data for patients categorised according to clinical or demographic characteristics other than age, such as sex, ethnicity, insulin delivery method, glycaemic control and depression comorbidity were very limited, with data available from only one or two studies. As with patient stratification by age, only data on DKA prevalence were available for the other categorical variables reviewed. Based on these limited data, higher prevalence of DKA was observed for women versus men, non-white versus white ethnicities, depressed versus non-depressed patients, patients with fair/poor versus excellent glycaemic control, and patients treated with insulin injections compared with those using CSII (figure 3B). ${ }^{11} 293637$

In a single study designed to investigate cross-sectional associations between patient characteristics and DKA events, ${ }^{11}$ female patients had a higher prevalence of DKA over the previous 12-month period than male patients 
( 55 vs 40 cases per 1000 people, respectively). This study also reported a prevalence of DKA in white non-Hispanic patients of 43 per 1000, while all other ethnicities had considerably higher prevalence of DKA, ranging from 81 per 1000 (other race/ethnicity) to 121 per 1000 (black non-Hispanic) during the same study period. ${ }^{11}$ Higher prevalence of DKA was observed among depressed patients (110 per 1000 for patients with at least one DKA event in the previous 3 months) than non-depressed patients (40 per 1000 for patients with at least one DKA event in the previous 3 months). ${ }^{29}$

DKA was more prevalent in patients with fair or poor glycaemic control, defined as HbA1c $\geq 8.5 \%$ (120 per 1000 for patients with at least one DKA event in the previous 12 months) ${ }^{37}$ In contrast, the lowest prevalence of DKA was reported for patients with excellent glycaemic control, defined as HbA1c $<6.5 \%$ (10 per 1000 for patients with at least one DKA event in the prior 12 months). ${ }^{37}$ Patients treated with CSII had lower prevalence of DKA than did patients using injectable insulin. ${ }^{1136}$ This trend was seen across multiple age groups in one study. ${ }^{36}$ However, duration of treatment with a CSII may affect prevalence of DKA, as data for patients who had recently (within the prior year) initiated pump therapy had similar rates of DKA to participants treated with insulin injections (both groups had 54 events per 1000), and the lower DKA prevalence (43 events per 1000) was observed only in patients who had been treated with a CSII for at least the previous year. ${ }^{11}$ It should be noted that these numerical trends did not demonstrate a statistically significant difference between insulin delivery groups in this study.

\section{DKA risk factors and associations}

Over half of the included studies (13 publications) reported at least some data on risk factors or patient characteristics associated with DKA events ${ }^{1120-2327-29} 3638404243$ (see online supplementary appendix 3). Almost all of these investigations used multiple regression analyses to evaluate the associations between baseline parameters and risk of DKA, adjusted for potential confounding factors such as age, sex, body mass index (BMI) and duration of diabetes. ${ }^{1120-23}$ 27-29 364042 Two of the studies that investigated risk factors associated with DKA only provided qualitative summaries of the associations. ${ }^{36} 43$

Several studies identified patient characteristics that were significantly associated with increased risk of DKA. ${ }^{112} 22233642$ The most frequently reported parameters correlating with DKA events were higher HbAlc/ poor glycaemic control, ${ }^{112} 22233642$ lower socioeconomic status (based on income, formal education and private insurance or some combination thereof), ${ }^{11} 202242$ depression/psychiatric symptoms or diagnosis at baseline 202829 and female sex. ${ }^{11} 2242$ Regarding the patient subgroups of interest, some conflicting data were presented for the relationship between DKA events and ethnicity or insulin delivery method. In a population restricted to young adults only (aged 18 years to 25 years) from the T1D Exchange Clinic Registry, Cengiz and colleagues ${ }^{22}$ found that both non-white race and use of MDIs (versus CSII) were significantly associated with an increased frequency of DKA events. ${ }^{22}$ In contrast, in a study examining a broader adult population (also from the T1D Exchange Clinic Registry), while non-white race was significantly associated with greater frequency of DKA events in a univariate analysis, after adjusting for socioeconomic status, non-white race was no longer a significant predictor of DKA. ${ }^{11}$ Similarly, this investigation ${ }^{11}$ found no difference in rates of DKA based on insulin delivery method.

\section{Quality assessment of included studies}

Regarding the quality of the studies included in this SLR, while each study did have potential limitations that should be considered when interpreting the results (table 1 for studies that reported incidence rates and table 2 for studies that reported prevalence), most investigations were scored as moderate quality based on an assessment using a standardised tool (the JBI prevalence studies quality assessment tool; see online supplementary appendix 4). Nearly all studies included in the SLR were susceptible to potential selection bias in the patient population evaluated. In many cases, this was due to use of a clinic-based registry (such as the T1D Exchange Clinic Registry or the DPV database), ${ }^{11} 22272933$ 36-38 40 which may not be representative of a broader population-based cohort of T1D patients; in addition, findings from investigations based on patients recruited from a single centre 232835394143 may not be generalisable to a wider group of patients with T1D. No studies were identified by this review that used an unselected population-based approach to recruit subjects, such as surveys based on population census data.

Many studies included in this SLR did not provide sufficient information to make a clear determination of study quality for some aspects of study design; this lack of detail was particularly notable for ascertainment of cases of T1D. Only two studies ${ }^{33} 42$ included any description of criteria for the diagnosis of T1D. Of these two, the Chinese study ${ }^{42}$ refers to American Diabetes Association and WHO guidelines for diagnosis but does not explicitly state the criteria used to determine T1D cases. Furthermore, many publications did not describe whether (or how) the included patient cohort differed from the broader population of adults with T1D, which makes an evaluation of potential selection bias, and generalisability, more difficult. When insufficient details were provided to permit assessment of a given study quality parameter, the study was given an 'unclear' rating for that aspect of study quality.

Regarding the definition or method of determination of DKA events, there was little consensus among the included studies. Several publications (for example, those based on the T1D Exchange Clinic Registry ${ }^{1122} 2933$ 36-38) relied on patient recall of past DKA events. Many studies evaluated DKA events as recorded in hospital/medical records (note that some of these studies used patient report of hospitalisation), ${ }^{11}$ 20-22 27-29 36-38 4042 while other investigations did not require hospitalisation as part of 


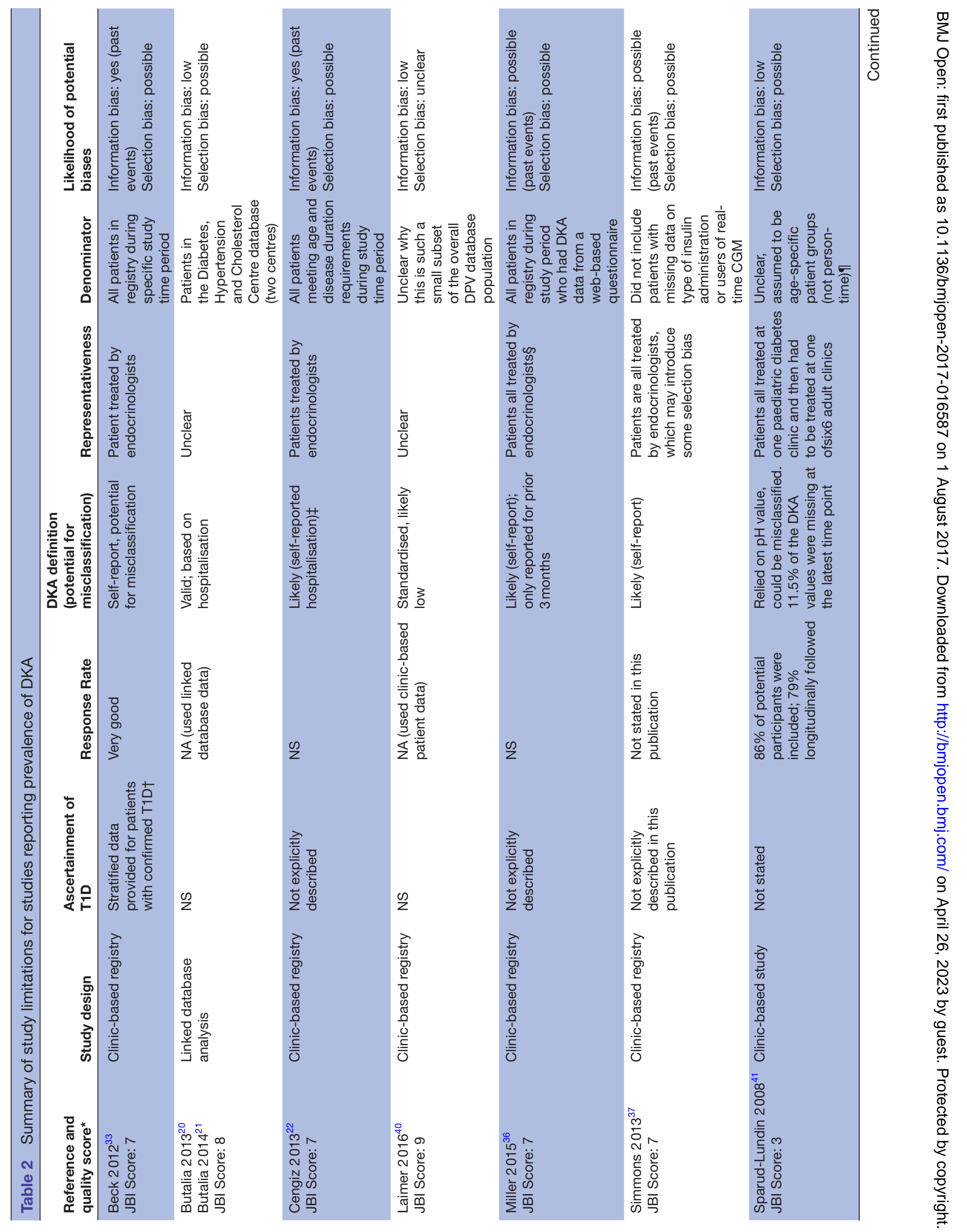




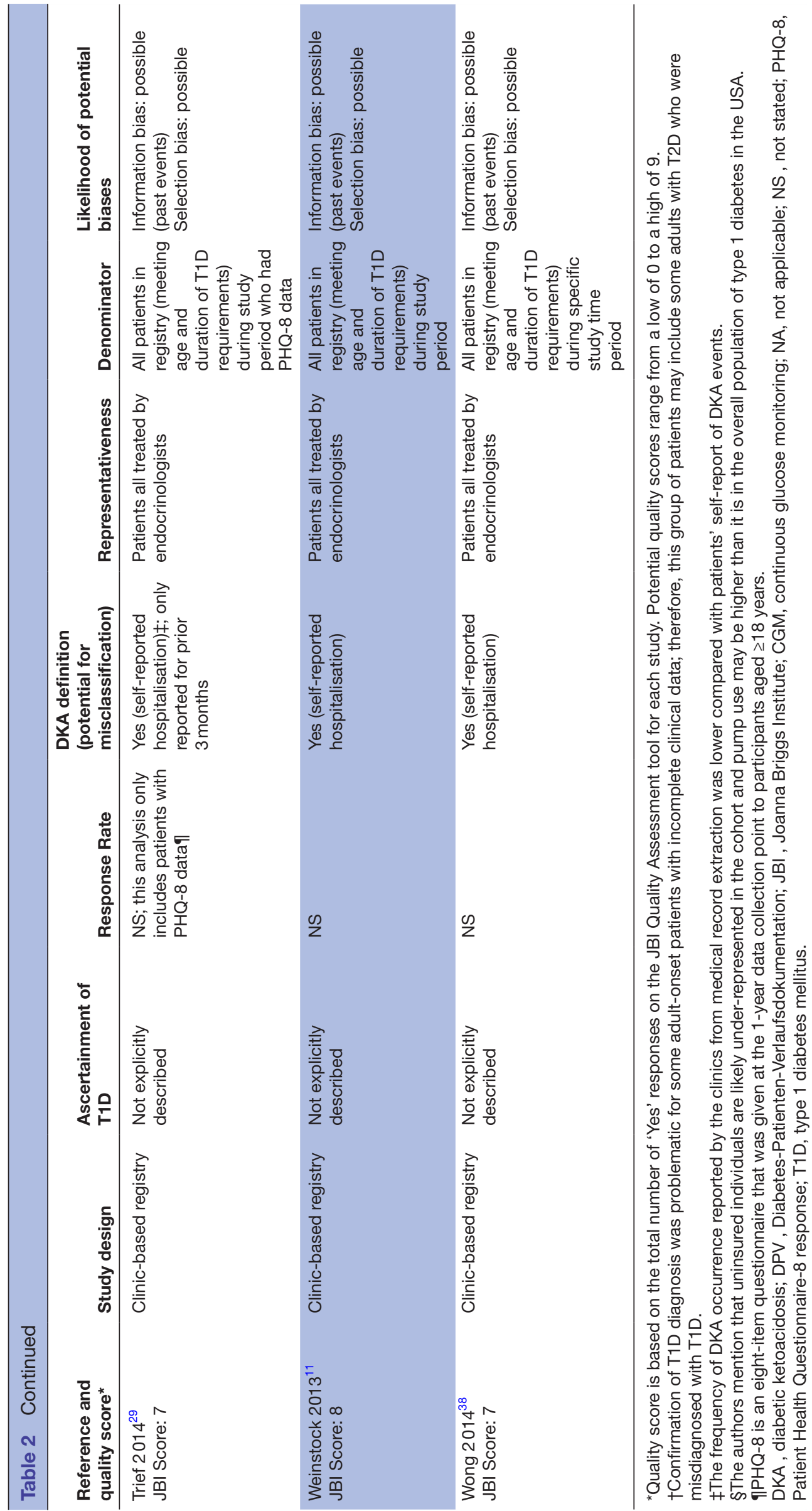


the definition of DKA or suggested the patient required intravenous fluid or insulin infusion without specifically stating a requirement for hospitalisation. ${ }^{23} 4143$ Interestingly, three publications ${ }^{1122} 29$ based on the T1D Exchange Clinic Registry did a direct comparison of frequency of DKA events based on patient self-report versus medical record extraction and, in each case, found that the number of events was higher for patient self-reporting than was captured in the patients' medical records. The authors suggested that DKA may be under-reported in clinical records and, therefore, chose to use patient self-reported data for further analyses.

While most studies were rated as having moderate study quality based on the JBI prevalence studies quality assessment checklist (see online supplementary appendix 4), a few outliers were identified with both high and low quality. Most of the studies that scored highly on the quality assessment ${ }^{1120212740}$ did so because they provided additional information and details not available in other publications. For example, a study of the DPV database evaluating the impact of physical activity on diabetes outcomes $^{27}$ reported a direct comparison of baseline characteristics of patients included in the analysis and those excluded due to missing data to rule out a significant impact of selection bias in this study. Similarly, as mentioned above, in Weinstock ${ }^{11}$ the authors included two sets of analyses using data for DKA events based on patient self-report and patients' medical records in an attempt to address the limitation of patient recall in determining the frequency of the DKA outcome. In contrast, studies that received lower quality ratings provided incomplete or conflicting information that made it difficult to evaluate the results. ${ }^{354}$ In a single-centre study conducted in Colorado (USA), ${ }^{35}$ ascertainment of T1D cases and definition of DKA were not reported, the study period and denominator for calculation of prevalence or incidence were unclear, and the sample size was relatively small (515 patients). Likewise, in a single-centre cohort study performed in Slovenia, ${ }^{39}$ the definitions for T1D cases, DKA events and denominator for determination of DKA events were not reported, and the study included only 184 patients. Given the limited data identified in the published literature on the epidemiology of DKA in adults with T1D, even studies that received lower quality ratings were included in this review, to present the totality of the available evidence. In online supplementary appendix 4, the notation 'Unclear' generally means that insufficient details were provided in the publication to make an informed determination of study quality for that particular question of the JBI assessment tool.

\section{DISCUSSION}

Of the 1082 citations identified, 19 publications met the inclusion and exclusion criteria for this SLR. Over half of the included studies evaluated patient cohorts based in North America ${ }^{1120-22} 29$ 33-38; data were more limited for studies conducted in Europe en 28 39-41 $^{2}$ elsewhere. ${ }^{2342} 43$ Overall, eight studies reported incidence rate, with a range of 0-263 per 1000 PYs, ${ }^{23} 27283435394243$ and 11 studies reported prevalence with a range of $0-128$ per 1000 people. $^{11} 20-222933$ 36-38 4041 The lowest incidence rates were reported in Israeli and North American studies $^{34} 43$ and the highest in a Chinese study. ${ }^{42}$ The lowest prevalence was reported in a Swedish study ${ }^{41}$ and the highest in a Canadian study. ${ }^{21}$ No publications reported both incidence rate and prevalence of DKA. Five studies ${ }^{20} 21283539$ provided sufficiently detailed information to allow for calculation of one of the outcomes of interest when these measures were not directly reported by the study authors. Several publications reported DKA outcome data stratified by age. ${ }^{112233} 3638$ In contrast, subgroup data for patients categorised based on other baseline characteristics, such as sex, ${ }^{11}$ ethnicity ${ }^{11}$ or insulin delivery method ${ }^{1136}$ were scarce. While there was considerable variation in study design and data sources among the studies included in the SLR, the majority of investigations presented recently obtained data (within the previous 10 years), and patient baseline characteristics were broadly similar. Many studies were cross-sectional in design or were identified as cross-sectional by the study authors, particularly those examining large $(>10000)$ patient databases 2227333638 ; the few studies that followed patients longitudinally tended to be single centre and to have small $(<200)$ sample sizes. ${ }^{28941}$ Based on the limited available data, prevalence and incidence rates for DKA were broadly similar across geographical regions but did differ for specific subgroups of patients.

Most studies included in this SLR were assessed as being of moderate quality. Nearly all studies in the review were susceptible to potential selection bias in the included patient population or were of limited generalisability. In addition, many included studies did not provide sufficient information to make a clear determination of quality for some aspects of study design; this lack of detail was particularly notable for ascertainment of cases of T1D. Furthermore, many publications did not describe whether (or how) the included patient cohort differed from the broader population of adults with T1D, which makes an evaluation of potential selection bias, and generalisability, more difficult.

There was little consensus among the included studies regarding the definition of, or method to determine, DKA events. One of the main issues affecting the quality determination for many of the included studies is the fact that the epidemiology of DKA events was not a primary (or, in many cases, even a secondary) objective of the study; rather, DKA data were reported only as part of overall rates of acute diabetic complications (along with other parameters such as severe hypoglycaemic events). This may contribute to the lack of detailed descriptions of DKA events. The findings from the Chinese study highlight the difficulties encountered in comparing the epidemiological data across the included studies, in which the methods of calculating incidence rate or prevalence often were not explicitly described. In particular, 
calculations of incidence rate are challenging without complete information on the patient numerator, given that a single patient can experience multiple recurrent DKA events; it is important to determine whether the incidence rate refers to the number of discrete episodes of DKA or to the number of patients who experienced a DKA event. Most studies ${ }^{1120-22293335-41}$ (13 of 19) reported the percentage of patients who had experienced at least one DKA episode (or two or more episodes ${ }^{28}$ ) over a given study period rather than the total number of DKA events. In other cases, data were aggregated as cumulative sums of DKA events during the study period ${ }^{27}$ or reported as events per patient per year, ${ }^{43}$ and some studies ${ }^{23} 344243$ did not provide details regarding the method of calculation of incidence rates.

To our knowledge, this is the first SLR on the epidemiology of DKA in adults with T1D. The strength of this study is the strict delineation that was taken to appropriately assess epidemiology data specifically in adults with T1D. Of note, both young adults and elderly patients were included in this SLR, so the results could be applicable across the whole spectrum of the adult population. Many (24) studies were omitted from the SLR based on the inability to stratify adult data separately from paediatric and/or adolescents or T1D data from a combined population of patients with T1D and T2D.

This review, like any SLR, is subject to limitations that should be considered when interpreting the results. All SLRs are subject to publication bias, as an SLR inherently relies on data available in the published literature. ${ }^{32}$ While a few studies were identified by this SLR that reported findings that did not support one of the authors' primary hypotheses (eg, in Butalia, ${ }^{21}$ driving distance to outpatient care was not associated with diabetic outcomes; in Wong, ${ }^{38}$ there was no significant association between use of continuous glucose monitoring and DKA events among adult patients with T1D), it is likely that null results may be infrequently published. In addition, only data from studies published in English were included in the SLR. This restriction may limit the available data from certain geographical regions in which English is not the primary language of publication and limits the overall scope of the review. As part of the abstract review process, the authors identified non-English studies (which had English abstracts available for review) and found fewer than 10 studies that had the potential for inclusion in the SLR, with data from Japan, China, Bulgaria, Senegal and the Ivory Coast. Similarly, studies of fewer than 50 adult patients with T1D were excluded. This restriction was deemed reasonable given the epidemiological outcomes of interest (prevalence and incidence rate), as deriving these values from a very small patient population would lead to a high degree of uncertainty in the estimates. However, it is likely that relevant data for smaller cohorts of patients may not have been included due to this restriction. This SLR was originally intended to include only population-based studies but was expanded to include clinic-based and (potentially unrepresentative) registry studies since there were so few population-based studies found. The small number of studies identified by the review limits the interpretation of comparisons within or between geographical regions and subgroups defined by patient clinical characteristics.

Of note, although the authors acknowledge the availability of nationwide population-based databases with high ascertainment rates in the Nordic countries, which could be used to evaluate epidemiological queries in T1D, publications on DKA rates among adults in this region were very limited; only one such study ${ }^{41}$ met the inclusion criteria for this SLR. Two additional studies of potential interest were identified but ultimately excluded from this review; an epidemiology study based on Denmark public health registries reported the incidence of DKA in the general population and not just among patients with T1D and was thus excluded from the SLR. ${ }^{44}$ A study from Sweden reported an incidence rate for DKA of 5.9 per 100000 people with T1D but defined adults as $\geq 15$ years of age; since this SLR strictly evaluated patients $\geq 18$ years of age, the study was excluded. ${ }^{45}$ Similarly, two additional publications ${ }^{46}{ }^{47}$ reporting data on DKA incidence or prevalence based on patients in the UK were excluded from this review due to lack of patient demographic information; neither study provided sufficient details to allow determination of the patient age range and therefore may have included data for paediatric T1D patients. Based on hospital records in Leicestershire, England ${ }^{46}$ the prevalence of DKA could be calculated as 13.7 per 1000 people over the 2-year study period. An investigation of patients with T1D in Scotland ${ }^{47}$ found that the cumulative incidence of DKA events was 154 events per 1000 people for the overall population, with considerable variation based on patients' economic status.

The wide range of incidence rates and prevalence of DKA in adults with T1D 2327283435394243 is similar to the published literature for children. The incidence of DKA in children with T1D (aged 0-18 years) was lowest in Sweden (15 per $1000 \mathrm{PY}$ ) and highest in the US (80-150 per 1000 PY; children aged $0-19$ years) prior to the Diabetes Control and Complications Trial (DCCT), based on a summary of the epidemiological literature at the time. ${ }^{48}$ After raised awareness associated with the DCCT, the incidence of DKA in children with T1D (aged 13-17 years) was 47 per 1000 PY with conventional therapy compared with 28 per 1000 PY with intensive therapy. ${ }^{48}$ Whereas adults with T1D have decreasing prevalence of DKA with increasing age, ${ }^{11}$ an opposite relationship may exist in children. In subgroup analyses of children with T1D, incidence of DKA increased with age for girls ( 40 per $1000 \mathrm{PY}$ in girls $<7$ years of age; 80 per 1000 PY in girls 7-12 years of age; 120 per 1000 PY in girls $\geq 13$ years of age, $p<0.001$ for trend) but not for boys (70 per 1000 PY in boys $<7$ years of age; 50 per 1000 PY in boys $7-12$ years of age; 80 per $1000 \mathrm{PY}$ in boys $\geq 13$ years of age). ${ }^{48}$ This suggests a plateau effect for risk of DKA, particularly in females. Rewers and colleagues ${ }^{48}$ indicated that the increased risk of DKA among adolescent girls (relative to younger children) may be related to body image issues that 
lead adolescent girls to skip insulin injections to promote weight loss. Increased insulin resistance due to puberty or obesity may also play a role in greater risk of DKA, as higher insulin dose was a predictor of DKA at all ages. Eating disorders, frequent among children with diabetes, also may affect risk of DKA but may be challenging to identify in this population. ${ }^{48}$ In one study using the Diabetes Audit and Research in Tayside Scotland database, it was suggested that poor adherence to insulin treatment in young adults with insulin-dependent diabetes mellitus is the major factor that contributes to long-term poor glycaemic control and diabetic ketoacidosis. ${ }^{49}$

Similar to adults with $\mathrm{T} 1 \mathrm{D},{ }^{11}$ the prevalence of DKA is higher in non-white versus white ethnicities in children. Non-Hispanic black children with T1D have the highest rate of DKA (23\%) compared with Hispanic children $(12 \%)$ and non-Hispanic white children $(7 \%) .{ }^{50}$ Also similar to adults, ${ }^{11}$ the risk of DKA increases in children with psychiatric disorders, those who are underinsured and those who have uncontrolled HbA1c. ${ }^{48}$

Given the above limitations of many of the available publications, there is a clear need for future investigations to better elucidate the epidemiology of DKA among adult patients with T1D. For future studies, it will be important to clearly describe how cases of T1D are identified and to use a standardised definition of DKA, as both of these factors are weaknesses of the currently available evidence. Ideally, future studies would focus specifically on DKA outcomes and employ population-based methods to identify patients with T1D and would therefore be more representative of a broad, unselected patient population. It would also be advisable to use data from some of the existing large, multicentred, clinic-based registries, such as the US-based T1D Exchange Clinic Registry, ${ }^{11} 22$ 29 33 36-38 Nordic databases, the Clinical Practice Research Datalink in the UK and the German/Austrian DPV ${ }^{27} 40$ to evaluate large cohorts of patients longitudinally to attempt to confirm some of the associations that have been suggested by cross-sectional analyses of these databases and to identify any changes in DKA trends over time. Since DKA is a recently recognised potential adverse event associated with some approved treatments for T2D, such as sodium-glucose cotransporter-2 inhibitors, and phase three trials are being conducted to determine the risk/benefit profile of the use of these therapies in patients with T1D, it would be prudent to better elucidate the expected background rate of DKA among adults with T1D. In addition, since DKA is a potentially life-threatening complication and there are currently limited data available on the mortality rates of DKA in a general T1D population, the existing large data sources in the USA and Europe could be used to describe DKA-related mortality.

\section{CONCLUSIONS}

This SLR is, to our knowledge, the first review to describe the epidemiology of DKA among adult patients with T1D. The review identified a limited number of relevant studies; most data were from clinic-based registries of selected patient populations, and most patient cohorts were based in North America. Patient subgroup data were very limited, but a general trend was observed for decreasing prevalence of DKA with increasing patient age. Several other factors, such as lower socioeconomic status, poor glycaemic control, female sex and depression or psychiatric symptoms, were associated with increased risk of DKA. There is a clear need for future studies to better describe the epidemiology of DKA among adult patients with T1D. From the currently available body of evidence, which provides an overall prevalence of DKA ranging from approximately 50 to 100 events per 1000 adult patients with $\mathrm{T} 1 \mathrm{D}$, it is clear that there remains an unmet need to address the prevention of this serious complication.

Acknowledgements The authors would like to thank LoAn K Ho, PharmD of Xcenda, LLC who provided medical writing services by preparing the first draft of the manuscript based on an existing report.

Contributors BAM, EW, SFF conceived and designed the study. EW and BAM undertook the literature search, assessed studies for eligibility and extracted data. In case of disagreement, SFF checked the study. BAM, EW, SFF, KB, NS and JM discussed the data, interpreted the results, reviewed the manuscript and revised it critically. NS and JM provided clinical input. All authors approved the final version and take full responsibility for the integrity of the data and the accuracy of the data summarisation and interpretation.

Funding This study was sponsored by Boehringer Ingelheim International GmbH. Competing interests All authors have completed the ICMJE uniform disclosure form at www.icmje.org/coi_disclosure.pdf and declare: SFF, KB, NS, and JM are employees of Boehringer Ingelheim. EW and BAM are employees of Xcenda, LLC.

Provenance and peer review Not commissioned; externally peer reviewed.

Data sharing statement Additional data are available in the appendices.

Open Access This is an Open Access article distributed in accordance with the Creative Commons Attribution Non Commercial (CC BY-NC 4.0) license, which permits others to distribute, remix, adapt, build upon this work non-commercially, and license their derivative works on different terms, provided the original work is properly cited and the use is non-commercial. See: http://creativecommons.org/ licenses/by-nc/4.0/

(C) Article author(s) (or their employer(s) unless otherwise stated in the text of the article) 2017. All rights reserved. No commercial use is permitted unless otherwise expressly granted.

\section{REFERENCES}

1. Centers for Disease Control and Prevention. National Diabetes Statistics Report: Estimates of Diabetes and Its Burden in the United States. 2014 www.cdc.gov/diabetes/pubs/statsreport14/nationaldiabetes-report-web.pdf.

2. Maahs DM, West NA, Lawrence JM, et al. Epidemiology of type 1 diabetes. Endocrinol Metab Clin North Am 2010;39:481-97.

3. International Diabetes Federation. IDF Diabetes Atlas . 7th Edition, 2015. Available from. www.diabetesatlas.org.

4. Patterson CC, Dahlquist GG, Gyürüs E, et al. EURODIAB Study Group. Incidence trends for childhood type 1 diabetes in Europe during 1989-2003 and predicted new cases 2005-20: a multicentre prospective registration study. Lancet 2009;373:2027-33.

5. Bruno G, De Salvia A, Arcari R, et al. Clinical, immunological, and genetic heterogeneity of diabetes in an Italian populationbased cohort of lean newly diagnosed patients aged 30-54 years. Piedmont Study Group for Diabetes Epidemiology. Diabetes Care 1999;22:50-5.

6. Diaz-Valencia PA, Bougnères P, Valleron AJ. Global epidemiology of type 1 diabetes in young adults and adults: a systematic review. BMC Public Health 2015;15:255. 
7. Frongia O, Mastinu F, Sechi GM. Prevalence and 4-year incidence of insulin-dependent diabetes mellitus in the province of Oristano (Sardinia, Italy). Acta Diabetol 1997;34:199-205

8. Lammi N, Taskinen O, Moltchanova E, et al. A high incidence of type 1 diabetes and an alarming increase in the incidence of type 2 diabetes among young adults in Finland between 1992 and 1996. Diabetologia 2007;50:1393-400.

9. Muntoni S, Songini M. High incidence rate of IDDM in Sardinia. Sardinian Collaborative Group for Epidemiology of IDDM. Diabetes Care 1992;15:1317-22.

10. Eledrisi MS, Alshanti MS, Shah MF, et al. Overview of the diagnosis and management of diabetic ketoacidosis. Am J Med Sci 2006;331:243-51.

11. Weinstock RS, Xing D, Maahs DM, et al. Severe hypoglycemia and diabetic ketoacidosis in adults with type 1 diabetes: results from the T1D Exchange clinic registry. J Clin Endocrinol Metab 2013;98:3411-9.

12. Nyenwe EA, Kitabchi AE. The evolution of diabetic ketoacidosis: an update of its etiology, pathogenesis and management. Metabolism 2016;65:507-21.

13. Agarwal A, Yadav A, Gutch M, et al. Prognostic factors in patients hospitalized with Diabetic Ketoacidosis. Endocrinology and metabolism 2016;31:424-32.

14. Gibb FW, Teoh WL, Graham J, et al. Risk of death following admission to a UK hospital with diabetic ketoacidosis. Diabetologia 2016;59:2082-7.

15. Kitabchi AE, Umpierrez GE, Miles JM, et al. Hyperglycemic crises in adult patients with diabetes. Diabetes Care 2009;32:1335-43.

16. Dahlquist G, Källén B. Mortality in childhood-onset type 1 diabetes: a population-based study. Diabetes Care 2005;28:2384-7.

17. Laing SP, Swerdlow AJ, Slater SD, et al. The British Diabetic Association Cohort Study, II: cause-specific mortality in patients with insulin-treated diabetes mellitus. Diabet Med 1999;16:466-71.

18. Patterson CC, Dahlquist G, Harjutsalo V, et al. Early mortality in EURODIAB population-based cohorts of type 1 diabetes diagnosed in childhood since 1989. Diabetologia 2007;50:2439-42.

19. Realsen J, Goettle H, Chase HP. Morbidity and mortality of diabetic ketoacidosis with and without insulin pump care. Diabetes Technol Ther 2012;14:1149-54.

20. Butalia S, Johnson JA, Ghali WA, et al. Clinical and sociodemographic factors associated with diabetic ketoacidosis hospitalization in adults with type 1 diabetes. Diabet Med 2013;30:567-73.

21. Butalia S, Patel AB, Johnson JA, et al. Association between diabetic ketoacidosis hospitalizations and driving distance to outpatient diabetes centres in adults with type 1 diabetes mellitus. Can $J$ Diabetes 2014;38:451-5.

22. Cengiz E, Xing D, Wong JC, et al. T1D Exchange Clinic Network. Severe hypoglycemia and diabetic ketoacidosis among youth with type 1 diabetes in the T1D Exchange clinic registry. Pediatr Diabetes 2013;14:447-54.

23. Lebenthal Y, Shalitin S, Yackobovitch-Gavan M, et al. Retrospective comparative analysis of metabolic control and early complications in familial and sporadic type 1 diabetes patients. J Diabetes Complications 2012;26:219-24.

24. Cheng YC, Huang $\mathrm{CH}$, Lin WR, et al. Immunology. Clinical outcomes of septic patients with diabetic ketoacidosis between 2004 and 2013 in a tertiary hospital in Taiwan. Journal of microbiology, 2014.

25. Hanas R, Ludvigsson J. Hypoglycemia and ketoacidosis with insulin pump therapy in children and adolescents. Pediatr Diabetes 2006; 7(Suppl 4):32-8

26. Phillip $\mathrm{M}$, Battelino $\mathrm{T}$, Rodriguez $\mathrm{H}$, et al.Use of insulin pump therapy in the pediatric age-group: consensus statement from the European Society for Paediatric Endocrinology, the Lawson Wilkins Pediatric Endocrine Society, and the International Society for Pediatric and Adolescent Diabetes, endorsed by the American Diabetes Association and the European Association for the Study of Diabetes. Diabetes Care 2007;30:1653-62.

27. Bohn B, Herbst A, Pfeifer M, et al. DPV Initiative. Impact of physical activity on Glycemic Control and prevalence of Cardiovascular Risk factors in adults with type 1 Diabetes: a Cross-sectional Multicenter Study of 18,028 patients. Diabetes Care 2015;38:1536-43.

28. Bryden KS, Dunger DB, Mayou RA, et al. Poor prognosis of young adults with type 1 diabetes: a longitudinal study. Diabetes Care 2003;26:1052-7.
29. Trief PM, Xing D, Foster NC, et al. T1D Exchange Clinic Network. Depression in adults in the T1D Exchange Clinic Registry. Diabetes Care 2014;37:1563-72.

30. Usher-Smith JA, Thompson M, Ercole A, et al. Variation between countries in the frequency of diabetic ketoacidosis at first presentation of type 1 diabetes in children: a systematic review. Diabetologia 2012;55:2878-94.

31. Zayed H. Epidemiology of diabetic ketoacidosis in Arab patients with type 1 diabetes: a systematic review. Int J Clin Pract 2016;70:186-95.

32. Moher D, Liberati A, Tetzlaff J, et al. PRISMA Group. Preferred reporting items for systematic reviews and meta-analyses: the PRISMA statement. PLoS Med 2009;6:e1000097.

33. Beck RW, Tamborlane WV, Bergenstal RM, et al. T1D Exchange Clinic Network. The T1D Exchange clinic registry. J Clin Endocrinol Metab 2012;97:4383-9.

34. Nathan DM, Zinman B, Cleary PA, et al.Modern-day clinical course of type 1 diabetes mellitus after 30 years' duration: the diabetes control and complications trial/epidemiology of diabetes interventions and complications and Pittsburgh epidemiology of diabetes complications experience (1983-2005). Arch Intern Med 2009;169:1307-16.

35. Garg SK, Walker AJ, Hoff HK, et al. Glycemic parameters with multiple daily injections using insulin glargine versus insulin pump. Diabetes Technol Ther 2004:6:9-15.

36. Miller KM, Foster NC, Beck RW, et al. Current state of type 1 diabetes treatment in the U.S.: updated data from the T1D Exchange clinic registry. Diabetes Care 2015;38:971-8.

37. Simmons JH, Chen V, Miller KM, et al. Differences in the management of type 1 diabetes among adults under excellent control compared with those under poor control in the T1D Exchange Clinic Registry. Diabetes Care 2013;36:3573-7.

38. Wong JC, Foster NC, Maahs DM, et al. Real-time continuous glucose monitoring among participants in the T1D Exchange clinic registry. Diabetes Care 2014;37:2702-9.

39. Janez A. Continuous subcutaneous insulin infusion in adult type 1 diabetes mellitus: data from a registry at the University Medical Centre Ljubljana, Slovenia. J Int Med Res 2012;40:1546-51.

40. Laimer M, Melmer A, Mader JK, et al. Variability of basal rate profiles in insulin pump therapy and association with complications in type 1 Diabetes Mellitus. PLoS One 2016;11:e0150604.

41. Sparud-Lundin C, Ohrn I, Danielson E, et al. Glycaemic control and diabetes care utilization in young adults with type 1 diabetes. Diabet Med 2008;25:968-73.

42. Li J, Yang D, Yan J, et al. Guangdong Type 1 Diabetes Translational Study Group. Secondary diabetic ketoacidosis and severe hypoglycaemia in patients with established type 1 diabetes mellitus in China: a multicentre registration study. Diabetes Metab Res Rev 2014;30:497-504.

43. Shalitin S, Lahav-Ritte T, Lebenthal Y, et al. Does the timing of insulin pump therapy initiation after type 1 diabetes onset have an impact on glycemic control? Diabetes Technol Ther 2012;14:389-97.

44. Henriksen OM, Røder ME, Prahl JB, et al. Diabetic ketoacidosis in Denmark incidence and mortality estimated from public health registries. Diabetes Res Clin Pract 2007;76:51-6.

45. Wang ZH, Kihl-Selstam E, Eriksson JW. Ketoacidosis occurs in both type 1 and type 2 diabetes--a population-based study from Northern Sweden. Diabet Med 2008;25:867-70.

46. Dave J, Chatterjee S, Davies M, et al. Evaluation of admissions and management of diabetic ketoacidosis in a large teaching hospital. Practical Diabetes International 2004;21:149-53.

47. Govan L, Maietti E, Torsney B, et al. The effect of deprivation and $\mathrm{HbA} 1 \mathrm{c}$ on admission to hospital for diabetic ketoacidosis in type 1 diabetes. Diabetologia 2012;55:2356-60.

48. Rewers A, Chase HP, Mackenzie T, et al. Predictors of acute complications in children with type 1 diabetes. JAMA 2002;287:2511-8.

49. Morris AD, Boyle DIR, McMahon AD, et al. Adherence to insulin treatment, glycaemic control, and ketoacidosis in insulin-dependent diabetes mellitus. The Lancet 1997;350:1505-10.

50. Willi SM, Miller KM, DiMeglio LA, et al. Racial-ethnic disparities in management and outcomes among children with type 1 diabetes. Pediatrics 2015;135:424-34. 
Correction: Incidence and prevalence of diabetic ketoacidosis

(DKA) among adults with type 1 diabetes mellitus (T1D): a systematic literature review

Fazeli Farsani S, Brodovicz K, Soleymanlou N, et al. Incidence and prevalence of diabetic ketoacidosis (DKA) among adults with type 1 diabetes mellitus (T1D): a systematic literature review. BMJ Open 2017;7:e016587. doi: 10.1136/bmjopen-2017-016587

The name of the corresponding author should be 'Fazeli Farsani S' instead of 'Farsani SF'.

Open Access This is an Open Access article distributed in accordance with the Creative Commons Attribution Non Commercial (CC BY-NC 4.0) license, which permits others to distribute, remix, adapt, build upon this work non-commercially, and license their derivative works on different terms, provided the original work is properly cited and the use is non-commercial. See: http://creativecommons.org/licenses/by-nc/4.0/

(C) Article author(s) (or their employer(s) unless otherwise stated in the text of the article) 2017. All rights reserved. No commercial use is permitted unless otherwise expressly granted.

BMJ Open 2017;7:e016587corr1. doi:10.1136/bmjopen-2017-016587corr1

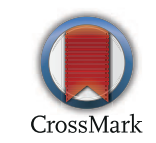

